\begin{tabular}{|c|c|c|}
\hline 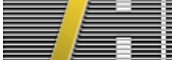 & 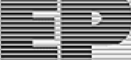 & PUBlished FOR SISSA BY 昜 SPRINGER \\
\hline & & $\begin{array}{l}\text { ReCEIVED: May 9, } 2015 \\
\text { ACCEPted: June 23, } 2015 \\
\text { Published: July 14, } 2015\end{array}$ \\
\hline
\end{tabular}

\title{
Comments on quantum Higgs vacua
}

\section{Jeffrey A. Harvey, David Kutasov and Sungjay Lee}

Enrico Fermi Institute and Department of Physics, University of Chicago, 5620 Ellis Av., Chicago Illinois 60637, U.S.A.

E-mail: jh25@uchicago.edu, dkutasov@uchicago.edu, sjlee79@uchicago.edu

ABSTRACT: We study two-dimensional $\mathcal{N}=(4,4)$ supersymmetric gauge theories which do not have classical Higgs branches, but are believed to have isolated quantum Higgs vacua. We provide arguments for the existence of such vacua based on brane dynamics in string theory and the supersymmetric partition sums of the gauge theories.

KeYwords: Supersymmetry and Duality, Supersymmetric gauge theory, D-branes

ARXiv EPRINT: 1406.6000 


\section{Contents}

1 Introduction 1

2 Brane perspective 2

$\begin{array}{llr}3 & \text { Supersymmetric index } & 6\end{array}$

\section{Introduction}

Many two dimensional supersymmetric gauge theories have a classical moduli space of vacua consisting of Higgs and Coulomb branches connected at the origin. ${ }^{1}$ Due to the large infrared fluctuations associated with scalar fields in two dimensions, one might expect the low energy theory to be a sigma model on this moduli space. Instead, it often splits into a direct sum of separate theories associated with the Coulomb and Higgs branches [1].

A simple example of this phenomenon occurs in $\mathcal{N}=(4,4)$ supersymmetric QED with $F$ charged hyper multiplets. Classically, this theory has a one (quaternionic) dimensional Coulomb branch and an $F-1$ dimensional Higgs branch, that intersect at the origin of both. Quantum mechanically, it splits in the infrared into two decoupled SCFT's, which correspond to the two classical branches and in general have different central charges.

From the perspective of the Coulomb branch theory the classical origin is pushed to infinity by a quantum correction to the metric. More precisely, the one-loop exact metric on the Coulomb branch is [2]

$$
d s^{2}=\left(\frac{1}{g^{2}}+\frac{F}{|\vec{\phi}|^{2}}\right) d \vec{\phi} \cdot d \vec{\phi},
$$

where $\vec{\phi}$ are the scalars in the $(4,4)$ vector multiplet, and $g^{2}$ is the gauge coupling constant. The origin of the Coulomb branch, $\vec{\phi}=0$, which classically is a finite point, lies quantum mechanically at the bottom of an infinite throat. This is regarded as a signal of the above decoupling in this branch [1]. It is also useful to recall for future reference that the Coulomb branch can be lifted by turning on an FI term $\xi$ for the U(1) factor in the gauge group, which breaks the gauge symmetry and leaves only the Higgs branch theory.

An interesting special case of the above theory is $F=1$, i.e., one charged hyper multiplet. For $\xi \neq 0$ this theory has an isolated Higgs vacuum in which the gauge symmetry is broken. For $\xi=0$, the classical analysis suggests that there is a Coulomb branch, but no Higgs vacuum. However, there are reasons to believe that there is actually a separate isolated Higgs vacuum which does not contain any light excitations and owes its existence

\footnotetext{
${ }^{1}$ In general there are also mixed branches; our discussion is easy to generalize to include them.
} 


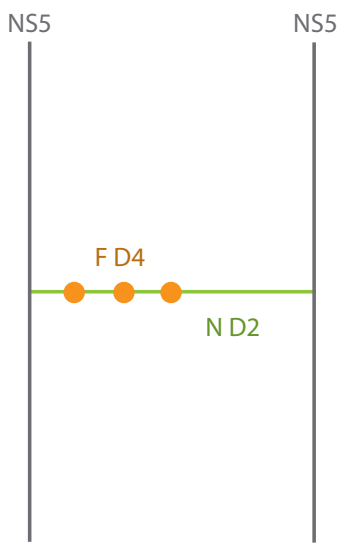

(a)

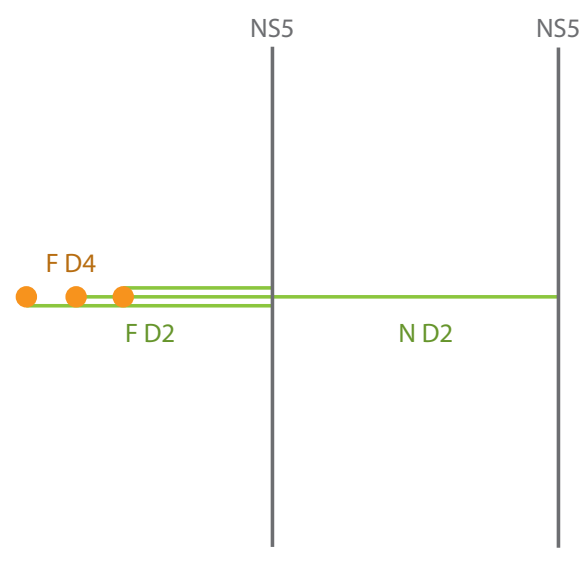

(b)

Figure 1. IIA brane realizations of $\mathcal{N}=(4,4)$ SQCD.

to quantum dynamics. This vacuum is important for describing fivebranes in matrix string theory, and for understanding the D1-D5 system.

A similar quantum Higgs vacuum is expected to appear in theories with higher rank gauge groups, such as $\mathcal{N}=(4,4)$ supersymmetric QCD with gauge group $\mathrm{U}(N)$ and $F=N$ hyper multiplets in the fundamental representation. One can think of its existence as a consequence of the duality satisfied by the Higgs branch theory, under which $(N, F) \rightarrow$ $(F-N, F)$.

In this note we discuss these vacua from two points of view: brane dynamics in string theory, and the supersymmetric index of the relevant gauge theory. In section 2 we review the embedding of $\mathcal{N}=(4,4)$ SQCD in string theory as the low energy theory on a system of intersecting NS5-branes and D-branes, which makes the quantum vacuum structure more transparent, partly because it makes the above Seiberg-like duality manifest. We use this description to understand the isolated quantum Higgs vacua, and discuss briefly the closely related problem of the behavior of the duality under mass deformations. In section 3 we review the definition of the supersymmetric index of $\mathcal{N}=(0,2)$ supersymmetric theories computed recently in $[3,4]$ using localization (see also [5]). We then explain how the supersymmetric index of these gauge theories can be used to deduce the existence of the isolated quantum vacua with mass gap.

\section{Brane perspective}

Systems of NS5-branes and D-branes in string theory are useful for studying the low energy behavior of many supersymmetric and non-supersymmetric vacua of SYM theories in various dimensions (see e.g. [6] for a review), and this is the case here as well. The brane system relevant for describing $\mathcal{N}=(4,4) \mathrm{SQCD}^{2}$ is depicted in figure $1(\mathrm{a})$. The $\mathcal{N}=(4,4)$

\footnotetext{
${ }^{2}$ A dimensional reduction of that of [7], which was used to study $\mathcal{N}=4$ supersymmetric gauge theories in $2+1$ dimensions.
} 
$\mathrm{U}(N)$ gauge multiplet lives on $N$ D2-branes stretched in the $x^{6}$ direction between two NS5branes whose world volume fills the (2345) directions. Both types of branes are extended in the $1+1$ dimensional spacetime labeled by (01). The hypermultiplets live at intersections of the D2-branes and F D4-branes stretched in (01789). To summarize, the worldvolume directions of the various branes are:

\begin{tabular}{|c|cc|cccc|c|ccc|}
\hline & 0 & 1 & 2 & 3 & 4 & 5 & 6 & 7 & 8 & 9 \\
\hline NS5 & $\times$ & $\times$ & $\times$ & $\times$ & $\times$ & $\times$ & & & & \\
D2 & $\times$ & $\times$ & & & & & $\times$ & & & \\
D4 & $\times$ & $\times$ & & & & & & $\times$ & $\times$ & $\times$ \\
\hline
\end{tabular}

To study the isolated quantum Higgs vacua it is convenient to use an alternative representation of this system, depicted in figure 1(b). The representations of figures 1(a) and (b) are related by the Hanany-Witten transition [6, 7]. Consider, for instance, the case $N=1$ (gauge group $\mathrm{U}(1)$ ). The classical Coulomb branch is parametrized by the location in the (2345) directions of the color D2-brane stretched between the fivebranes. The Higgs branch is obtained in figure 1(a) by letting the D2-brane split into segments on the D4-branes. The $F-1$ moduli correspond to the positions of the segments stretched between adjacent D4-branes in the (789) directions. ${ }^{3}$ In figure 1(b) the Higgs branch is described by reconnecting the color D2-brane to the longest flavor D2-brane, and allowing the segments of the resulting two brane connecting adjacent D4-branes to move in the (789) directions [6]. The two branches intersect at the origin of both, which is depicted in figure 1.

Turning on an FI term $\xi$ for the $\mathrm{U}(1)$ corresponds in the brane description to a relative displacement of the two NS5-branes in the transverse directions (789). As is clear from figure 1, this lifts the classical Coulomb branch, and leaves behind only the Higgs branch.

For $N=F=1$ we recover the structure mentioned in section 1 . For $\xi \neq 0$ we find a vacuum, which from the brane perspective is described by a configuration in which the color and flavor D2-branes in figure 1(b) combine into a single D2-brane that connects the right NS5-brane to the D4-brane, never intersecting the second NS5-brane. Since the low energy theory on such a D2-brane is massive [7], this vacuum is isolated.

For $\xi=0$, the classical picture of figure 1 suggests that the above isolated state becomes identical to the origin of the Coulomb branch, and in particular there is no independent isolated vacuum. However, as we mentioned, quantum mechanically we expect such a vacuum to exist. To understand this from the brane point of view we recall that the classical configurations of figure 1 are corrected at finite $g_{s}$ by brane bending effects $[8,9]$. A D2-brane ending on an NS5-brane can be thought of as a charged particle in the five dimensional gauge theory obtained by reducing the fivebrane theory on $x^{1}$ (or, equivalently, a string charged under the self-dual $B_{\mu \nu}$ field in the six dimensional IIA fivebrane theory). Thus, the gauge field on the fivebrane has a non-trivial profile $F_{0 r} \sim 1 / r^{3}$, where $r$ is the

\footnotetext{
${ }^{3}$ The fourth modulus in the multiplet can be identified with the 6 component of the gauge field on the D2-branes.
} 


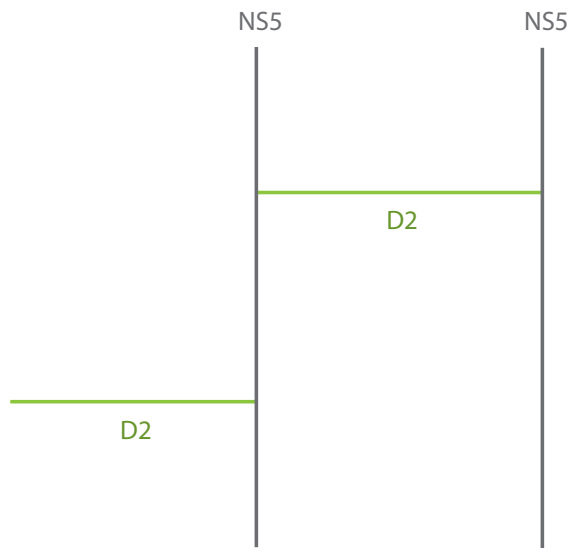

(a)

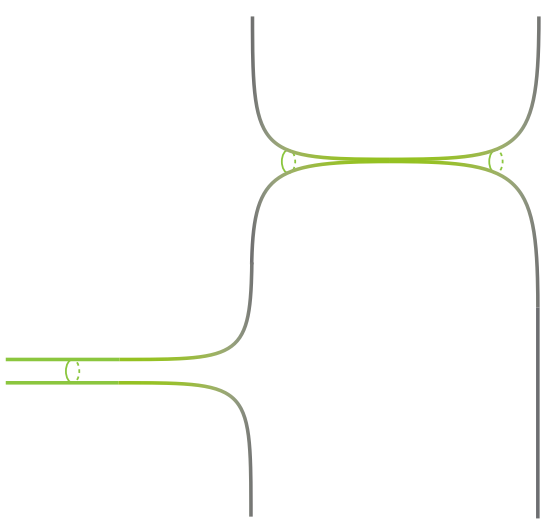

(b)

Figure 2. Classical (a) and quantum (b) descriptions of the Coulomb branch of $\mathrm{U}(1) \mathcal{N}=(4,4)$ SQED with one charged hyper multiplet.

radial direction in (2345). Since this configuration is BPS, there is also a non-trivial profile of the scalar field on the fivebrane $x^{6}(r) \sim 1 / r^{2}$.

This has an important effect on the vacuum structure. A generic point on the Coulomb branch, that classically is described by the configuration of figure 2(a), is replaced at finite $g_{s}$ by that of figure 2(b). In particular, the kinetic term for the position of the color D2-brane in the $(2345)$ directions, $\vec{\phi}\left(x^{\mu}\right)$, which classically is canonical, $\mathcal{L}_{\text {kin }} \sim\left|\partial_{\mu} \vec{\phi}\right|^{2}$, develops a singularity at $\vec{\phi}=0, \mathcal{L}_{\text {kin }} \sim\left|\partial_{\mu} \vec{\phi}\right|^{2} /|\vec{\phi}|^{2}$. Thus, the origin of the Coulomb branch is pushed to infinity, in agreement with the discussion of $[1,2]$. The supersymmetric brane configuration depicted in figure 3 represents the isolated quantum Higgs vacuum of the theory.

The above discussion easily generalizes to the case of $\mathrm{U}(N)$ gauge theory with $F=N$ hypermultiplets (see figure 1). It is obvious in the brane description that the classical theory (with $\xi=0$ ) has an $N$ dimensional Coulomb branch, parametrized by the positions of the $N$ color D2-branes, and no vacua with lower rank gauge group. Quantum mechanically, the vacuum structure is much richer: each of the $N$ color D2-branes can combine with a flavor D2-brane to form the configuration of figure 3, thereby reducing the rank of the gauge group by one unit. When all $N$ color D2-branes do this, one finds an isolated vacuum with no light excitations.

One can take a broader viewpoint and consider the theory with general $N$ and $F$ in figure 1. This theory has a rich vacuum structure that depends on $N, F$. For $F<N$, a Higgs branch does not exist, and if we turn on a non-zero FI parameter $\xi$ for the U(1), there is no supersymmetric vacuum. For $F>N$ there is a Higgs branch of the classical moduli space in which the gauge symmetry is fully broken. Its dimension is given by $N(F-N)$. This branch gives rise in the quantum theory to a CFT with central charge $c=6 N(F-N)$.

This CFT enjoys a duality $N \rightarrow F-N$, which is due to the fact that the Higgs branches of the $\mathrm{U}(N)$ and $\mathrm{U}(F-N)$ theories with $F$ flavors coincide. In the gauge theory 


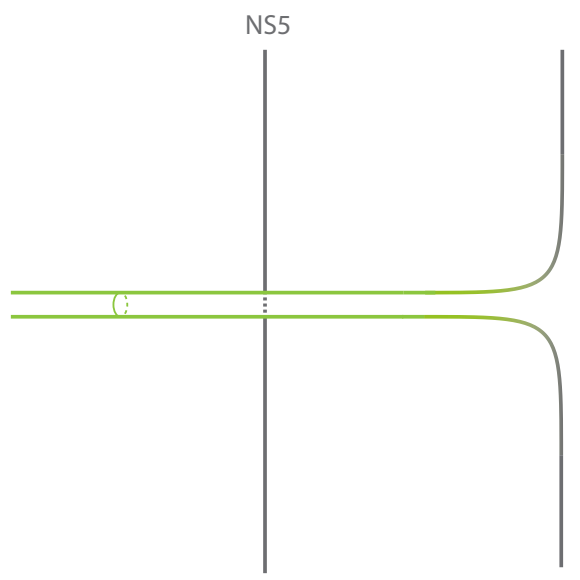

Figure 3. The quantum Higgs vacuum of the theory of figure 2 .

this is familiar from studies of $\mathcal{N}=2 \mathrm{SQCD}$ in four dimensions [10]. In two dimensions it was demonstrated that the two-sphere partition functions [11, 12] and elliptic genera [4] of these two gauge theories are the same, which supports the duality. In the brane language it is a simple generalization of the results of [13].

For $F=N$ the electric theory is the Higgs branch of the $\mathrm{U}(N)$ theory with $N$ flavors, while the magnetic theory becomes a $\mathrm{U}(0)$ theory, i.e. a supersymmetric vacuum with no light excitations. This supersymmetric vacuum is the isolated Higgs vacuum we discussed above.

We note in passing that the set of isolated vacua becomes richer when we consider deformations. For example, consider turning on (equal) masses for $F_{1}$ of the $F$ fundamental hypermultiplets. In the brane language this corresponds to displacing $F_{1}$ of the D4-branes from the origin of the (2345) space to a particular point while leaving the remaining $F_{2}=$ $F-F_{1}$ D4-branes at the origin. In order for the theory to have a Higgs branch, we need to divide the D2-branes into two groups, of $N_{1}$ and $N_{2}$ with $N_{1}+N_{2}=N$, and displace them so that they intersect the corresponding sets of D4-branes; see figure 4 for a simple example. The existence of a Higgs vacuum implies that $0 \leq N_{i} \leq F_{i}$, which can be equivalently written as

$$
\max \left(F_{1}-\hat{N}, 0\right) \leq N_{1} \leq \min \left(F_{1}, N\right)
$$

where $\hat{N}=F-N$. It is easy to see that this condition is invariant under the duality map $N \rightarrow \hat{N}, N_{i} \rightarrow \hat{N}_{i}=F_{i}-N_{i}$, in agreement with the fact that the low energy theory reduces in this case to a direct product of CFT's at the two intersections of $N_{i}$ and $F_{i}$ branes, with central charges $c_{i}=6 N_{i}\left(F_{i}-N_{i}\right)$.

Whenever one of the $N_{i}$ coincides with the corresponding $F_{i}$, we get an isolated vacuum in that theory, which corresponds under the duality map to a theory with no light excitations but a supersymmetric vacuum. Thus, the existence of the quantum Higgs vacua is necessary for the consistency of the $(4,4)$ duality with deformations. 


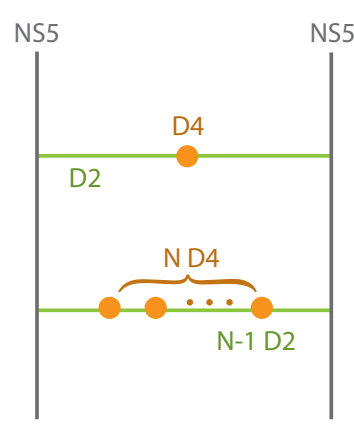

II

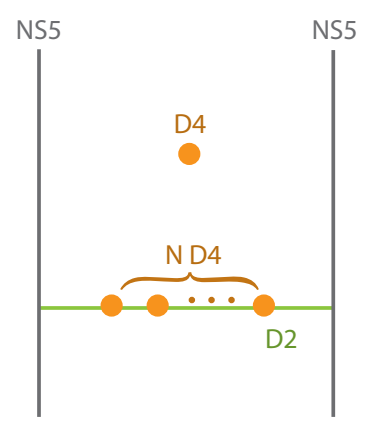

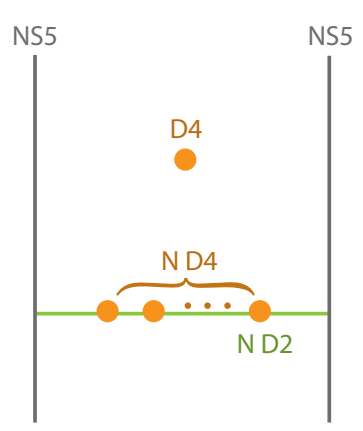

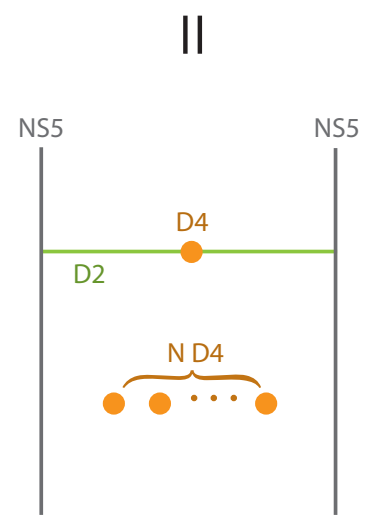

Figure 4. Map of Higgs vacua of a $\mathrm{U}(N)$ gauge theory with $F=N+1$ flavors (top) and the dual $\mathrm{U}(1)$ theory with $N+1$ flavors (bottom) when one of the flavors has a non-zero mass.

\section{Supersymmetric index}

Two dimensional $\mathcal{N}=(4,4) \mathrm{U}(N)$ SQCD coupled to $F$ hypermultiplets in the fundamental representation has a global $\mathrm{SU}(F)$ flavor symmetry, as well as an $\mathrm{SU}(2)_{1} \times \mathrm{SU}(2)_{2} \times \mathrm{SU}(2)_{3}$ R-symmetry, under which the vector multiplet scalars transform as $(\mathbf{1}, \mathbf{2}, \mathbf{2})$ and the hyper multiplet scalars transform as $(\mathbf{2}, \mathbf{1}, \mathbf{1})$. The four right-moving supercharges $\mathcal{Q}_{+}^{a \alpha}$ transform as $(\mathbf{2}, \mathbf{2}, \mathbf{1})$, while the four left-moving supercharges $\mathcal{Q}_{-}^{a \dot{\alpha}}$ transform as $(\mathbf{2}, \mathbf{1}, \mathbf{2})$. The indices $\alpha, \dot{\alpha}$ correspond to doublets under $\mathrm{SU}(2)_{2}$ and $\mathrm{SU}(2)_{3}$, respectively. For $F>N$, when the Higgs branch theory is non-trivial, $\mathrm{SU}(2)_{2}$ and $\mathrm{SU}(2)_{3}$ become parts of the infrared right and left-moving $\mathcal{N}=4$ superconformal algebras, respectively.

Choosing particular supercharges $\mathcal{Q}_{+}^{++}$and $\mathcal{Q}_{+}^{--}$that generate an $\mathcal{N}=(0,2)$ subalgebra of the full $\mathcal{N}=(4,4)$ SUSY algebra (i.e. $\left\{\mathcal{Q}_{+}^{++}, \mathcal{Q}_{+}^{--}\right\}=\bar{L}_{0}$ ), we can define a supersymmetric partition sum as follows

$$
\mathcal{E}\left(\tau ; z, \zeta_{i}\right)=\operatorname{Tr}_{\mathcal{H}_{\mathrm{RR}}}\left[(-1)^{F} q^{L_{0}} \bar{q}^{\bar{L}_{0}} e^{-2 \pi i z J_{R}} e^{-2 \pi i \zeta_{1}\left(J_{1}-J_{2}\right)} e^{-2 \pi i \zeta_{2} \cdot J_{f}}\right],
$$

where $\left\{J_{1}, J_{2}, J_{R}, J_{f}\right\}$ are Cartan subalgebra generators of the R-symmetry group $\mathrm{SU}(2)_{1} \times$ $\mathrm{SU}(2)_{2} \times \mathrm{SU}(2)_{3}$ and the flavor group $\mathrm{SU}(F)$. Note that the global charges $J_{R}, J_{1}-J_{2}$ and $J_{f}$ commute with the chosen supercharges $\mathcal{Q}_{+}^{++}$and $\mathcal{Q}_{+}^{--}$. Thus, states with $\bar{L}_{0} \neq 0$ come in pairs whose contributions to the partition sum (3.1) vanish. When the Higgs theory is 
non-trivial (for $F>N$ ), one can think of this partition sum as the (equivariant) elliptic genus of this theory, but it can be defined when the infrared SCFT is gapped and trivial as well, and we will in fact be interested in this case below. Thus, we will refer to (3.1) as a supersymmetric index.

The index (3.1) is independent of the FI parameter $\xi$, since the FI term is $\mathcal{Q}_{+}$-exact with $\mathcal{Q}_{+}=\mathcal{Q}_{+}^{++}+\mathcal{Q}_{+}^{--}$and crucially the asymptotic behavior of the potential on field space is independent of this parameter. In particular, the superficially flat potential on the Coulomb branch at $\xi=0$ is lifted by the nonzero background gauge field that couples to $J_{1}-J_{2}$.

As mentioned above, for $\xi \neq 0$ the Coulomb branch is lifted, so in that case the index must come from the Higgs branch. In particular, for the case $F=N$ where the vacuum is isolated (i.e. all field theoretic degrees of freedom are massive), the index must be equal to 1 ,

$$
\mathcal{E}\left(\tau ; z, \zeta_{i}\right)=1
$$

This index was recently computed in $[4,5]$ using localization. Below we will use their results to verify (3.2)

For $\xi=0$, we saw in the previous section that the infrared limit of the gauge theory with $F=N$ is a direct sum of the $N$ dimensional Coulomb branch, mixed branches in which the rank of the unbroken subgroup of $\mathrm{U}(N)$ is lower than $N$, and the isolated Higgs vacuum. The index is the sum of the contributions of the various branches. The $\xi$ independence of the index (3.1) implies that the result (3.2) must still be valid when $\xi=0$, and it is natural to ask which branch(es) it is coming from. We will see later that the Coulomb and mixed branches do not contribute to the index. Hence, the result (3.2) must come from the conjectured isolated Higgs vacuum. We conclude that this result requires the existence of the quantum Higgs branch with no massless excitations.

The above statements must be true on general grounds, but it is useful to verify them using the formalism of [4]. It is convenient to rewrite the index (3.1) in the form

$$
\mathcal{E}(\tau ; \vec{z})=\operatorname{Tr}_{\mathcal{H}_{\mathrm{RR}}}\left[(-1)^{F} q^{L_{0}} \bar{q}^{\bar{L}_{0}} e^{2 \pi i \vec{z} \cdot \vec{J}}\right]
$$

where $\vec{J}=\left(J_{R}, J_{1}-J_{2}, J_{f}\right)$. The authors of [4] showed that for any $\mathcal{N}=(0,2)$ supersymmetric gauge theory one can write $\mathcal{E}$ as

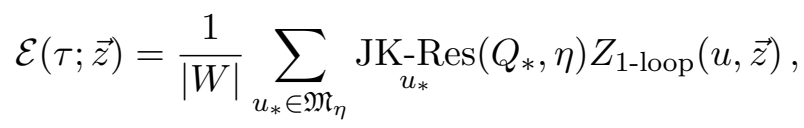

where $u$ denotes the holomorphic coordinates parametrizing the moduli space of flat gauge connection on the torus, $u \in(\mathbb{C} / \mathbb{Z}+\tau \mathbb{Z})^{r}$ for a rank $r$ gauge group, and $|W|$ is the order of the Weyl group. The one-loop determinant $Z_{1-\text { loop }}$ is

$$
Z_{1 \text {-loop }}(u)=Z_{\mathrm{vec}}(u) \cdot Z_{\mathrm{chi}}(u) \cdot Z_{\mathrm{fer}}(u)
$$


with the contributions from $(0,2)$ vector, chiral and Fermi multiplets given by

$$
\begin{aligned}
& Z_{\text {vec }}(u)=\left[\frac{2 \pi \eta(\tau)^{2}}{i}\right]^{r} \prod_{a: \text { roots }} \frac{i \vartheta_{1}(\tau, \alpha \cdot u)}{\eta(\tau)}, \\
& Z_{\text {chi }}(u)=\prod_{\rho: \text { weights }} \frac{i \eta(\tau)}{\vartheta_{1}\left(\tau, \rho \cdot u+\vec{z} \cdot \vec{J}_{c}\right)}, \\
& Z_{\text {fer }}(u)=\prod_{\rho: \text { weights }} \frac{i \vartheta_{1}\left(\tau, \rho \cdot u+\vec{z} \cdot \vec{J}_{f}\right)}{\eta(\tau)},
\end{aligned}
$$

where $\vec{J}_{c}$ and $\vec{J}_{f}$ are the $\vec{J}$ charges of chiral and Fermi multiplets. Finally, 'JK-Res' is the Jeffrey-Kirwan residue whose definition we review next.

Each $\vartheta_{1}$ factor in the denominator of $Z_{1 \text {-loop }}(3.6)$ defines a hyperplane in the $u$-plane,

$$
\rho \cdot u+\vec{z} \cdot \overrightarrow{J_{c}}=0
$$

where $Z_{1 \text {-loop }}(u)$ becomes singular. Such a hyperplane is associated with a charge vector $\rho$ in $\mathbb{R}^{r}$. Let us denote by $\mathfrak{M}$ the set of singular points $u_{*}$ where $n \geq r$ hyperplanes intersect. We focus on the case $n=r$, which is the case of interest for the theories discussed below. The Jeffrey-Kirwan residue depends on a choice of an $r$-component vector $\eta$. For given $\eta$, one can define a set $\mathfrak{M}_{\eta}$ of singular points in $\mathfrak{M}$ where $r$ hyperplanes intersect and the corresponding $r$ charge vectors generate a positive cone containing the vector $\eta$,

$$
\eta \in \operatorname{Cone}\left[\rho_{1}, \rho_{2}, \ldots, \rho_{r}\right] .
$$

In the case of $n=r$, the Jeffrey-Kirwan residue is defined as follows

$$
\underset{u_{*}}{\operatorname{JK} \operatorname{Res}} \frac{1}{\left(\rho_{1} \cdot u\right) \cdots\left(\rho_{r} \cdot u\right)}= \begin{cases}\frac{1}{\left|\operatorname{det}\left(\rho_{1} \cdots \rho_{r}\right)\right|} & \text { if } u_{*} \in \mathfrak{M}_{\eta} \\ 0 & \text { otherwise. }\end{cases}
$$

For more details of the Jeffrey-Kirwan residue see [4, 14]. The index is of course independent of the choice of $\eta$. For rank one gauge group with $\eta>0(\eta<0)$, the index $\mathcal{E}(\tau, \vec{z})$ becomes the sum of residues of $Z_{1 \text {-loop }}(u)$ at the poles $u=u_{*}$ associated with the fields of positive (negative) charge.

As a warm-up exercise, let us start with a U(1) theory with a single charged hyper multiplet of charge +1 . From (3.6) one can show that the one-loop factor corresponding to the $(4,4)$ vector multiplet is

$$
Z_{\mathrm{vec}}(u)=\frac{i \eta(q)^{3}}{\vartheta_{1}\left(\tau, \zeta_{1}-z\right)} \frac{\vartheta_{1}\left(\tau, 2 \zeta_{1}\right)}{\vartheta_{1}\left(\tau, \zeta_{1}+z\right)}
$$

while the contribution from the charged hyper multiplet is

$$
Z_{\text {hyper }}(u)=\frac{\vartheta_{1}(\tau, u-z)}{\vartheta_{1}\left(\tau, u-\zeta_{1}\right)} \frac{\vartheta_{1}(\tau,-u-z)}{\vartheta_{1}\left(\tau,-u-\zeta_{1}\right)} .
$$


Choosing $\eta>0$, the index thus becomes

$$
\mathcal{E}(\tau ; \xi, z)=\operatorname{Res}_{u=\zeta_{1}} Z_{\mathrm{vec}}(u) \cdot Z_{\mathrm{hyper}}(u)=1
$$

One can further show that the contribution to the index from the Coulomb branch vanishes. The low-energy theory on the Coulomb branch can be described by two $(0,2)$ neutral chiral multiplets $\Sigma, \tilde{\Sigma}$ and two neutral Fermi multiplets $\Upsilon, \tilde{\Upsilon}$. Here the Fermi multiplet $\Upsilon$ contains the field strength $F_{01}$. The global charges are given by $\vec{J}_{\Sigma}=(-1,1)$, $\vec{J}_{\tilde{\Sigma}}=(1,1), \vec{J}_{\Upsilon}=(0,0)$ and $\vec{J}_{\tilde{\Upsilon}}=(2,0)$. Since the Fermi multiplet $\Upsilon$ is neutral under both $J_{R}$ and $J_{1}-J_{2}$, one can show from (3.6) that the contribution from the Coulomb branch to the index vanishes. This is essentially because the fermionic zero mode in $\Upsilon$ will pair states of opposite fermion number and equal charges $\vec{J}$.

We next turn to the case of general $N$, i.e., $\mathcal{N}=(4,4) \mathrm{U}(N)$ gauge theory with $F=N$ fundamental hyper multiplets. We use the standard unit vectors $e_{i}$ in $\mathbb{R}^{N}, i=1,2, \ldots, N$, to parameterize the weight vectors of the fundamental representation. The root vectors can be expressed as $e_{i}-e_{j}$ with $i \neq j$. The one-loop determinant is in this case

$$
\begin{aligned}
Z_{\mathrm{vec}}(u)= & {\left[\frac{i \eta(q)^{3}}{\vartheta_{1}\left(\tau, \zeta_{1}-z\right)} \frac{\vartheta_{1}\left(\tau, 2 \zeta_{1}\right)}{\vartheta_{1}\left(\tau, \zeta_{1}+z\right)}\right]^{N} } \\
& \times \prod_{i \neq j} \frac{\vartheta_{1}\left(\tau,\left(e_{i}-e_{j}\right) \cdot u\right)}{\vartheta_{1}\left(\tau,\left(e_{i}-e_{j}\right) \cdot u+\zeta_{1}-z\right)} \frac{\vartheta_{1}\left(\tau,\left(e_{i}-e_{j}\right) \cdot u+2 \zeta_{1}\right)}{\vartheta_{1}\left(\tau,\left(e_{i}-e_{j}\right) \cdot u+\zeta_{1}+z\right)},
\end{aligned}
$$

and

$$
Z_{\text {hyper }}(u)=\prod_{i} \prod_{\mu: \text { weights }} \frac{\vartheta_{1}\left(\tau, e_{i} \cdot u+\mu \cdot \zeta_{2}-z\right)}{\vartheta_{1}\left(\tau, e_{i} \cdot u+\mu \cdot \zeta_{2}-\zeta_{1}\right)} \frac{\vartheta_{1}\left(\tau,-e_{i} \cdot u-\mu \cdot \zeta_{2}-z\right)}{\vartheta_{1}\left(\tau,-e_{i} \cdot u-\mu \cdot \zeta_{2}-\zeta_{1}\right)},
$$

where $\mu$ denote the weight vectors of the fundamental representations under the flavor group $\mathrm{SU}(N)$.

To compute the index,${ }^{4}$ we first choose the $N$-component vector $\eta$ as follows

$$
\eta=e_{1}+e_{2}+\ldots+e_{N} .
$$

One can show that any set of charge vectors generating a positive cone that contains the vector $\eta$ should have either a pair of weight vector $e_{i}$ and root vector $e_{j}-e_{i}$ for certain $i$ and $j$ or weight vectors only.

In the case that the set of charge vectors have a pair of $e_{i}$ and $e_{j}-e_{i}$, the Jeffrey-Kirwan residue becomes trivial. This is because the corresponding singular points satisfy

$$
\begin{array}{r}
\left(e_{j}-e_{i}\right) \cdot u_{*}+\zeta_{1} \pm z=0 \\
e_{i} \cdot u_{*}+\mu \cdot \zeta_{2}-\zeta_{1}=0
\end{array}
$$

with a weight vector $\mu$ in the fundamental representation of $\mathrm{SU}(N)$. This implies that

$$
e_{j} \cdot u_{*}+\mu \cdot \zeta_{2} \pm z=0
$$

for which one of the factors in the numerator of $Z_{\text {hyper }}$ in (3.14) vanishes.

\footnotetext{
${ }^{4}$ Our analysis is parallel to that of [4].
} 
For the cone generated by the weight vectors $\left\{e_{1}, e_{2}, \ldots, e_{N}\right\}$, there are corresponding $N^{N}$ singular points satisfying

$$
\begin{array}{r}
e_{1} \cdot u_{*}+\mu_{1} \cdot \zeta_{2}-\zeta_{1}=0, \\
e_{2} \cdot u_{*}+\mu_{2} \cdot \zeta_{2}-\zeta_{1}=0, \\
\vdots \\
e_{N} \cdot u_{*}+\mu_{N} \cdot \zeta_{2}-\zeta_{1}=0,
\end{array}
$$

for arbitrary $N$ weight vectors $\mu_{i}$. However if two such weight vectors coincide, for instance $\mu_{1}=\mu_{2}$, the Jeffrey-Kirwan residue vanishes because

$$
\left(e_{1}-e_{2}\right) \cdot u_{*}=0
$$

at which the factor $\vartheta_{1}\left(\tau,\left(e_{1}-e_{2}\right) \cdot u\right)$ in the numerator of $Z_{\text {vec }}$ in (3.13) becomes zero. Thus we have $N$ ! singular points with $\mu_{i} \neq \mu_{j}$ for any $i, j$, at each of which the Jeffrey-Kirwan residue can be shown to give one. Dividing by the order of the Weyl group (3.4) one finds

$$
\mathcal{E}\left(\tau ; z, \xi_{i}\right)=1
$$

As in the $\mathrm{U}(1)$ case, one can verify that the contributions to the index from the Coulomb and mixed branches vanish due to the existence of the neutral Fermi multiplet. Therefore, one concludes that there must exist an isolated quantum Higgs vacuum in the $\mathcal{N}=(4,4) \mathrm{U}(N)$ theory coupled to $N$ flavors.

\section{Acknowledgments}

We thank Piljin Yi for helpful discussions. JH acknowledges the support of NSF grant 1214409. DK is supported in part by DOE grant DE-FG02-13ER41958. The work of SL is supported in part by the Ernest Rutherford fellowship of the Science and Technology Facilities Council ST/J003549/1.

Open Access. This article is distributed under the terms of the Creative Commons Attribution License (CC-BY 4.0), which permits any use, distribution and reproduction in any medium, provided the original author(s) and source are credited.

\section{References}

[1] E. Witten, On the conformal field theory of the Higgs branch, JHEP 07 (1997) 003 [hep-th/9707093] [INSPIRE].

[2] M.R. Douglas, J. Polchinski and A. Strominger, Probing five-dimensional black holes with D-branes, JHEP 12 (1997) 003 [hep-th/9703031] [INSPIRE].

[3] F. Benini, R. Eager, K. Hori and Y. Tachikawa, Elliptic genera of two-dimensional $N=2$ gauge theories with rank-one gauge groups, Lett. Math. Phys. 104 (2014) 465 [arXiv: 1305.0533] [INSPIRE]. 
[4] F. Benini, R. Eager, K. Hori and Y. Tachikawa, Elliptic genera of $2 D \mathcal{N}=2$ gauge theories, Commun. Math. Phys. 333 (2015) 1241 [arXiv:1308.4896] [INSPIRE].

[5] A. Gadde and S. Gukov, 2D index and surface operators, JHEP 03 (2014) 080 [arXiv: 1305.0266] [INSPIRE].

[6] A. Giveon and D. Kutasov, Brane dynamics and gauge theory, Rev. Mod. Phys. 71 (1999) 983 [hep-th/9802067] [INSPIRE].

[7] A. Hanany and E. Witten, Type IIB superstrings, BPS monopoles and three-dimensional gauge dynamics, Nucl. Phys. B 492 (1997) 152 [hep-th/9611230] [INSPIRE].

[8] C.G. Callan and J.M. Maldacena, Brane death and dynamics from the Born-Infeld action, Nucl. Phys. B 513 (1998) 198 [hep-th/9708147] [InSPIRE].

[9] E. Witten, Solutions of four-dimensional field theories via M-theory, Nucl. Phys. B 500 (1997) 3 [hep-th/9703166] [INSPIRE].

[10] I. Antoniadis and B. Pioline, Higgs branch, hyperKähler quotient and duality in SUSY N $=2$ Yang-Mills theories, Int. J. Mod. Phys. A 12 (1997) 4907 [hep-th/9607058] [INSPIRE].

[11] F. Benini and S. Cremonesi, Partition functions of $\mathcal{N}=(2,2)$ gauge theories on $S^{2}$ and vortices, Commun. Math. Phys. 334 (2015) 1483 [arXiv:1206.2356] [InSPIRE].

[12] N. Doroud, J. Gomis, B. Le Floch and S. Lee, Exact results in D $=2$ supersymmetric gauge theories, JHEP 05 (2013) 093 [arXiv: 1206.2606] [INSPIRE].

[13] S. Elitzur, A. Giveon and D. Kutasov, Branes and $N=1$ duality in string theory, Phys. Lett. B 400 (1997) 269 [hep-th/9702014] [INSPIRE].

[14] K. Hosomichi and S. Lee, Self-dual strings and 2D SYM, JHEP 01 (2015) 076 [arXiv: 1406.1802] [INSPIRE]. 